\title{
Sox9 Is Crucial for Mesenchymal Stem Cells to Enhance Cutaneous Wound Healing
}

\author{
Qingli Bie ${ }^{1,3}$, Ruixia Zhai ${ }^{2}$, Yanrong Chen ${ }^{1}$, Yingao $\mathrm{Li}^{1}, \mathrm{Na} \mathrm{Xie}^{1}$, Baoyi Wang ${ }^{3}$, Poyun Yuan ${ }^{3}$, \\ Xinjie Zhou ${ }^{3}$, Haiyan Cong, ${ }^{4}$ Xin Chang ${ }^{4}$, Huabao Xiong ${ }^{5}$, Bin Zhang ${ }^{1,3}$ \\ ${ }^{I}$ Department of Laboratory Medicine, Affiliated Hospital of Fining Medical University, Fining Medical University, Fining, China \\ ${ }^{2}$ Department of Obstetric, Affiliated Hospital of Fining Medical University, Fining Medical University, Fining, China \\ ${ }^{3}$ Institute of Forensic Medicine and Laboratory Medicine, Fining Medical University, Fining, China \\ ${ }^{4}$ Department of Central Lab, Weihai Municipal Hospital, Cheeloo College of Medicine, Weihai, China \\ ${ }^{5}$ Institute of Immunology and Molecular Medicine, Fining Medical University, fining, China
}

Background and Objectives: Human umbilical cord mesenchymal stem cells (HUC-MSCs) are promising candidates for cell-based therapy in regenerative medicine or other diseases due to their superior characteristics, including higher proliferation, faster self-renewal ability, lower immunogenicity, a noninvasive harvest procedure, easy expansion in vitro, and ethical access, compared with stem cells from other sources.

Methods and Results: In the present study, we knocked down the expression of SOX9 in HUC-MSCs by lentivirus interference and found that knockdown of SOX9 inhibited the proliferation and migration of HUC-MSCs and influenced the expression of cytokines (IL-6 and IL-8), growth factors (GM-CSF and VEGF) and stemness-related genes (OCT4 and SALL4). In addition, the repair effect of skin with burn injury in rats treated with HUC-MSCs transfected with sh-control was better than that rats treated with HUC-MSCs transfected with shSOX9 or PBS, and the accessory structures of the skin, including hair follicles and glands, were greater than those in the other groups. We found that knockdown of the expression of SOX9 obviously inhibited the expression of Ki67, CK14 and CK18.

Conclusions: In conclusion, this study will provide a guide for modifying HUC-MSCs by bioengineering technology in the future.

Keywords: HUC-MSCs, SOX9, Skin, Regenerative medicine

Received: April 20, 2021, Revised: June 9, 2021,

Accepted: July 8, 2021, Published online: August 31, 2021

Correspondence to Bin Zhang

Department of Laboratory Medicine, Affiliated Hospital of Jining

Medical University, 89 Guhuai Road, Jining 272000, China

Tel: +86-0537-3616505, Fax: +86-0537-2903223

E-mail: zhb861109@163.com

Co-Correspondence to Huabao Xiong

Institute of Immunology and Molecular Medicine, Jining Medical

University, 133 HehuaRoad, Jining 272000, China

Tel: +86-0537-3616505, Fax: +86-0537-2903223

E-mail: xionghbl@yahoo.com

(a) This is an open-access article distributed under the terms of the Creative Commons Attribution Non-Commercial License (http://creativecommons.org/ licenses/by-nc/4.0/), which permits unrestricted non-commercial use, distribution, and reproduction in any medium, provided the original work is properly cited.

Copyright (c) 2021 by the Korean Society for Stem Cell Research

\section{Introduction}

Mesenchymal stem cells (MSCs) are nonhematopoietic cells that were first isolated from mouse bone marrow by Friedenstein (1). Studies have revealed that MSCs are multipotent cells with the potential to self-renew and differentiate into various cell types, including osteocytes, chondrocytes, adipocytes, and cells of other embryonic lineages (2). MSCs have been successfully isolated from many adult tissues, including cord blood (3), adipose tissue (4), skin (5), umbilical cord (6), amniotic membrane (7) and even the fetal lung and liver $(8,9)$. Owing to the self-renewal and multilineage differentiation of MSCs, these cells have therapeutic potential in tissue healing and 
regenerative medicine.

The human umbilical cord is a promising source of mesenchymal stem cells. HUC-MSCs have a painless collection procedure compared with mesenchymal stem cells from other sources and are noncontroversial sources compared to embryonic stem cells (10). HUC-MSCs are an optimal choice for stem cell-based therapy due to their superior characteristics, including less stringent requirements for HLA matching, reduced graft versus host disease (GVHD), improved access to transplantation and easy expansion in vitro (11). Jiang et al. (12) reported the first clinical trial describing the application of HUC-MSCs in 2011 and assessed the safety and efficiency of HUC-MSCs by treating four patients with stroke. Several studies have reported the application of HUC-MSCs in kidney injury (13), neurological deficits (14), immune system diseases (15), diabetes (16), and acute leukemia (17). A variety of phase I or II clinical therapeutic trials of HUC-MSCs on spinal cord injury, cerebral hemorrhage, Alzheimer's disease, hereditary ataxias, liver failure, graft-versus-host disease, type 2 diabetes, and rheumatoid arthritis have been completed (18).

Cutaneous wounds are very common and have high mortality and morbidity (19); these wounds destroy the barrier function of the skin and alter the perceptions of pain, temperature and touch (20). Our previous report showed that HUC-MSC-exosome-derived Wnt4 protein enhances skin cell proliferation and migration by activating $\beta$-catenin signaling and promotes wound healing in the early phase in a rat model of deep second-degree burn injury (21). We found that HUC-MSC exosome-delivered 14-3-3 $\zeta$ orchestrates self-control of the Wnt response by regulating YAP during the late phase of cutaneous regeneration (22). Our two reports collectively demonstrated that HUC-MSC-exosomes function not only as an "accelerator" of the Wnt/ $\beta$-catenin signal to repair damaged skin tissue but also as a "brake" of the signal by regulating YAP to control cutaneous regeneration $(21,22)$. In addition, we demonstrated that HUC-MSC exosomes enhance angiogenesis of damaged skin tissue (23).

SOX9 is a member of the sex-determining region $\mathrm{Y}$ (SRY)-related high mobility group (HMG)-box (SOX) transcription factor family (24). SOX9, an early embryonic development-related gene, plays a critical role in the development of cartilage and the testes (25). Research has shown that ectoderm- and endoderm-derived tissues continue to express SOX9 in stem cell pools (26), and evidence has also demonstrated that SOX9 participates in regulating cancer stem cells (CSCs) $(27,28)$. Several studies have revealed that decreasing the expression of SOX9 enhances the adipogenic potential of AML-MSCs and enhances their ability to support AML progenitor cells (29) and that adenoviral SOX9 gene transfer effectively induces chondrogenic differentiation of human primary MSCs in pellet culture (30). Elena Della Bella also demonstrated that dexamethasone induces osteogenic differentiation of human bone marrow mesenchymal stromal cells by inhibiting SOX9 expression (31). To date, studies on the effects of SOX9 on MSCs have mainly focused on their differentiation abilities. Paracrine components, including exosomes, cytokines, and growth factors, are important for the functions of MSCs. Our previous reports found huc-MSCs derived exosomes enhanced cutaneous wound healing in rat skin burn model $(21,22)$. Whether SOX9 regulates the functions and paracrine activity of HUC-MSCs has not been clarified to date.

In the present study, we found that knockdown of SOX9 expression in HUC-MSCs by lentivirus interference significantly decreased the proliferation and migration of HUC-MSCs and decreased the expression of inflammatory cytokines, VEGF and stemness-related genes. More importantly, knockdown of the expression of SOX9 in HUC-MSCs obviously decreased the ability to promote repair of skin tissue and healing. This study provides theoretical information for modifying HUC-MSCs by means of biological technology to strengthen the functions of HUC-MSCs in regenerative medicine.

\section{Materials and Methods}

\section{HUC-MSC isolation culture}

Human umbilical cord was collected from puerperants who underwent childbirth at the Affiliated Hospital of Jining Medical University (Jining, China). HUCMSCs were isolated and characterized as described previously (32). The cells at passages 3 and 4 were used for experiments. HUCMSCs were cultured in low-glucose Dulbecco's modified Eagle's medium (DMEM, HyClone) supplemented with $10 \%$ fetal bovine serum (FBS, Gibco) under standard conditions $\left(37^{\circ} \mathrm{C}\right.$ and $5 \% \mathrm{CO}_{2}$ in a humidified incubator).

\section{Ethics statement}

The study conformed to the principles outlined in the Declaration of Helsinki and was conducted in accordance with approved guidelines. Written informed consent was obtained from each participant prior to the collection of umbilical cord. All samples were acquired in accordance with the regulations and approval of the Medical Ethics Committee of Jining Medical University. 


\section{Lentiviral knockdown of Sox9 in HUC-MSCs cells}

The SOX9 shRNA sequence was synthesized and inserted into the shRNA expression vector pGPH1/Neo (GenePharma, Shanghai, China), and a scramble shRNA was used as a negative control (shNC). Cells were transfected with shSOX9 or shNC using Lipofectamine 2000 (Invitrogen) according to the manufacturer's instructions. Stable cell lines were obtained after selection with $1 \mu \mathrm{g} / \mathrm{ml}$ puromycin (Invitrogen) for 15 days. The expression of shRNA was induced by the addition of $80 \mu \mathrm{g} / \mathrm{ml}$ doxycycline.

\section{Cell proliferation experiments}

For the Cell Counting Kit-8 (CCK-8) assay, HUC-MSCs transfected with either sh-control or sh-Sox9 were incubated at $37^{\circ} \mathrm{C}$. CCK-8 solution (Dojindo, Japan) was then added to each well and incubated for $2 \mathrm{~h}$. Absorbance at $450 \mathrm{~nm}$ was measured at 12, 24, 36, and $48 \mathrm{~h}$.

For cell counting, HUC-MSCs (5,000/well) transfected with either sh-control or sh-Sox 9 were seeded in 24 plates and incubated in a $37^{\circ} \mathrm{C}$ and $5 \% \mathrm{CO}_{2}$ incubator. HUC-MSCs were counted continuously for 5 days, and cell proliferation was evaluated.

\section{Colony formation assay}

HUC-MSCs transfected with either sh-control or sh-Sox9 were harvested and seeded in $35-\mathrm{mm}$ plates $(1,000$ cells/well) overnight under standard conditions. Cells were incubated at $37^{\circ} \mathrm{C}$ in a $5 \% \mathrm{CO}_{2}$ humidified incubator for 10 days. At the end of the incubation period, the cultures were fixed with $4 \%$ paraformaldehyde and stained with crystal violet.

\section{Transwell migration assays}

HUC-MSCs transfected with either sh-control or sh-Sox $9\left(1 \times 10^{5}\right.$ cells/well $)$ were added to the top chamber, and cells that migrated through the $8-\mu \mathrm{m}$ pores and adhered to the lower surface of the membrane were fixed with $4 \%$ paraformaldehyde and then stained with crystal violet. Six random visual fields were observed and counted under a light microscope.

\section{RNA extraction and real-time RT-PCR (mRNA)}

Total RNA was extracted from cells using RNA isolate (Vazyme Biotech Co., Ltd.), and equal amounts of RNA were used for real-time RT-PCR analyses. The cDNAs were synthesized by using a reverse transcription kit according to the manufacturer's instructions (Vazyme Biotech Co., Ltd.). Real-time quantitative RT-PCR (qRT-PCR) was conducted with ChamQ Universal SYBR qPCR Master Mix (Vazyme Biotech Co., Ltd). $\beta$-actin was used as an internal control. The sequences of the specific primers are listed in Table 1.

\section{Rat skin wound model and treatment}

Adult female Sprague-Dawley rats (weighing $220 \pm 20 \mathrm{~g}$ ) were purchased from Jinan Pengyue Experimental Animal Breeding. A rat model of skin deep second-degree burn wounds was established as described previously (21). Rats were anesthetized with sodium thiopental at a dose of 40 $\mathrm{mg} / \mathrm{kg}$ body weight. After the hair on their upper back was shaved, the back skin of rats was injured with $80^{\circ} \mathrm{C}$ water

Table 1. Sequences of real-time PCR primers

\begin{tabular}{|c|c|c|c|}
\hline mRNA & Primer & Sequences $\left(5^{\prime}-3^{\prime}\right)$ & Annealing temperature \\
\hline \multirow[t]{2}{*}{ Human-IL-8 } & Forward & AGCTCTGTGTGAAGGTGCAG & $60^{\circ} \mathrm{C}$ \\
\hline & Reverse & ТСТСАGСССТСТТСАААААСТTС & \\
\hline \multirow[t]{2}{*}{ Human-IL-6 } & Forward & TTACAGGGAGAGGGAGCGAT & $60^{\circ} \mathrm{C}$ \\
\hline & Reverse & тTCTCTTTCGTTCCCGGTGG & \\
\hline \multirow[t]{2}{*}{ Human-GM-CSF } & Forward & TGGCCATGGAAAAGGGACTG & $63^{\circ} \mathrm{C}$ \\
\hline & Reverse & ACTGAGAGGCTGGTCCATCAGA & \\
\hline \multirow[t]{2}{*}{ Human-VEGF } & Forward & GTGACACCAGCTGTCTCCG & $60^{\circ} \mathrm{C}$ \\
\hline & Reverse & ACCCGTTGATCAGCAGAAGG & \\
\hline \multirow[t]{2}{*}{ Human-OCT4 } & Forward & TTGAGGCTCTGCAGCTTAG & $60^{\circ} \mathrm{C}$ \\
\hline & Reverse & GCCGGTTACAGAACCACAC & \\
\hline \multirow[t]{2}{*}{ Human-SALL4 } & Forward & TCGATGGCCAACTTCCTTC & $62^{\circ} \mathrm{C}$ \\
\hline & Reverse & GAGCGGACTCACACTGGAGA & \\
\hline LV3-homo-SOX9 & & GCATCCTTCAATTTCTGTATA & \\
\hline LV3NC & & TTCTCCGAACGTGTCACGT & \\
\hline \multirow[t]{2}{*}{ Human- $\beta$-actin } & Forward & GACCTGTACGCCAACACAGT & $59^{\circ} \mathrm{C}$ \\
\hline & Reverse & CTCAGGAGGAGCAATGATCT & \\
\hline
\end{tabular}


for 8 seconds to create a $16 \mathrm{~mm}$ diameter wound and then covered with gauze soaking saline for 6 minutes on the wound. Moreover, $1 \times 10^{8}$ cells (HUC-MSCs transfected with either sh-control or sh-Sox9) suspended in $200 \mu 1$ of PBS or $200 \mu 1$ of PBS were injected subcutaneously at three sites. The animals were housed individually. The healing area of the skin damage scar was photographed at $12,24,36$, and $48 \mathrm{~h}$. At 15 days after treatment, the rats were sacrificed, and the wound area was cut for further analysis.

\section{H\&E staining and immunofluorescence}

The wound skin $\left(4 \mathrm{~mm}^{2}\right)$ was fixed in $4 \%$ paraformaldehyde ( $\mathrm{pH} 7.4$ ), gradually dehydrated, embedded in paraffin, cut into $4-\mu \mathrm{m}$ sections and stained with $\mathrm{H} \& \mathrm{E}$ for light microscopy. The thickness of the epidermis and dermis layers was measured. Immunofluorescence staining was performed as described previously (33). Primary antibodies were as follows: CK14 (Bioworld), CK18 (Bioworld), and Ki67 (Cell Signaling Technology). Fluorescent secondary antibodies were obtained from Cell Signaling Technology. Images were sequentially acquired through light microscopy (Nikon, Tokyo, Japan) and confocal microscopy (GE, USA).

\section{Statistical analysis}

All data are shown as the mean \pm standard deviation (SD). The statistically significant differences between groups were assessed by analysis of variance (ANOVA) or t-test using Prism software (GraphPad, San Diego, USA). $\mathrm{p}$ values $<0.05$ were considered significant.

\section{Results}

\section{Knockdown of SOX9 expression decreased the proliferation and migration of HUC-MSCs}

Studies have revealed SOX9 regulates cancer stem-like properties and SLUG is required for SOX9 stabilization and functions to promote cancer stem cells $(27,28)$. And, SOX9 also regulates adipogenic, chondrogenic, osteogenic differentiation of mesenchymal stromal cells (29-31). So SOX9 is likely to be participated in regulating the regeneration roles of MSCs. To explore the influences of SOX9 on HUC-MSCs, we first isolated HUC-MSCs from the umbilical cord. Fig. 1 shows HUC-MSC growth from the tissue mass of the umbilical cord.

Wang et al. (34) have demonstrated that knockdown of SOX9 in triple-negative breast cancer cells increased apoptosis and the cell cycle was arrested at the G0/G1 phase, RNA sequencing identified numerous genes regu- lated by SOX9. Gao et al. (35) also revealed SOX9 downregulation decreased cyclin D1, CDK4 expression and $\mathrm{Rb}$ phosphorylation, which correlated with a reduced population of cells in the $S$ phase and suppressed growth of glioma cells. These reports revealed SOX9 could regulate the cell proliferation by influencing on cell cycle. We evaluated the effects of SOX9 on the proliferation of HUC-MSCs by Cell Counting Kit- 8 assays and continuous cell counting for 5 days and found that knockdown of SOX9 expression significantly decreased the proliferation of HUC-MSCs (Fig. 2A and 2B). A colony formation assay further confirmed this result; knockdown of SOX9 in HUC-MSCs significantly inhibited colony formation (Fig. 2C and 2D). MSCs possess a homing ability, ensuring that they can migrate to injured sites (36). Therefore, exploring the influences of SOX9 on the migration of HUC-MSCs is important for its application in regenerative medicine. We found that knockdown of SOX9 expression significantly attenuated the migration of HUC-MSCs (Fig. $2 \mathrm{E}$ and $2 \mathrm{~F}$ ). These results indicated that SOX9 plays critical roles in regulating the proliferation and migration of HUC-MSCs.

\section{The mRNA expression of cytokines and stemness-related genes in HUC-MSCs with SOX9 knockdown}

Interleukin (IL)-6 and interleukin (IL)-8, which are the most abundant cytokines secreted by MSCs, play critical roles in regulating tumor cell proliferation, tumor cell apoptosis, and tumorigenesis $(37,38)$. We detected the IL-6 and IL-8 mRNA expression in HUC-MSCs transfected with sh-control or shSOX9 by qRT-PCR and found that knockdown of SOX9 decreased IL-8 mRNA expression significantly and promoted IL-6 mRNA expression with no significant effect (Fig. 3A and 3B). Liu and Hwang (39) found that mesenchymal stem cells from
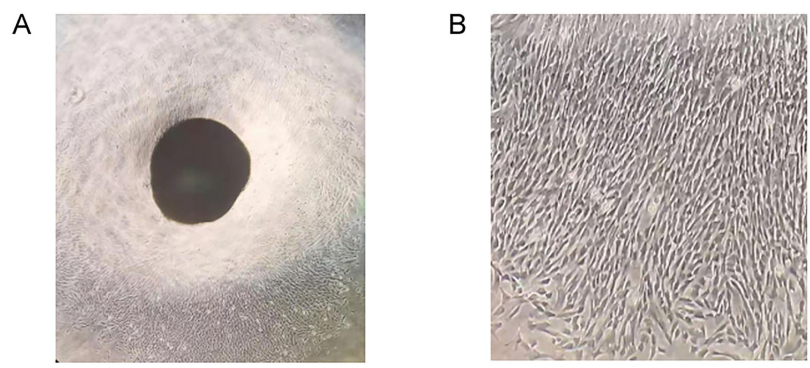

Fig. 1. Human umbilical cord mesenchymal stem cells. (A) HUC-MSCs growth from human umbilical cord tissue, which were observed on 3 4 days for culture by inverted microscope. (B) HUC-MSCs fusion reached $80 \%$ at one week of culture. 
A

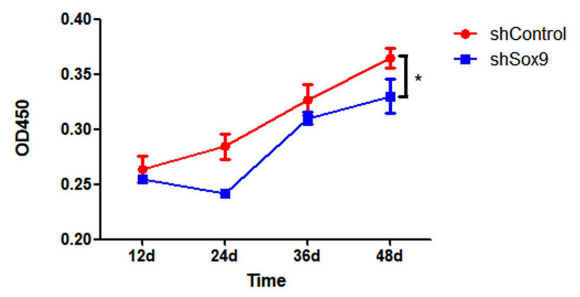

C

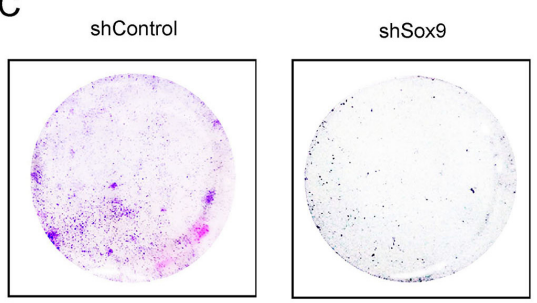

E shControl

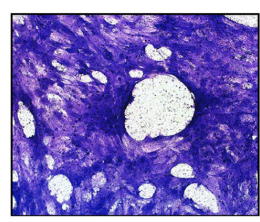

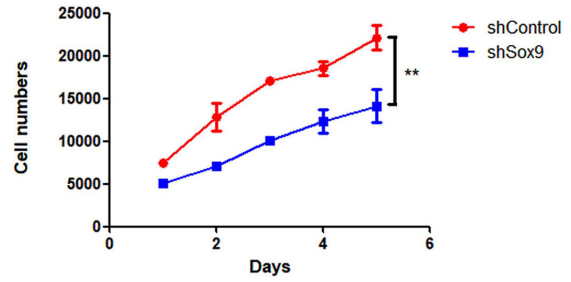

D

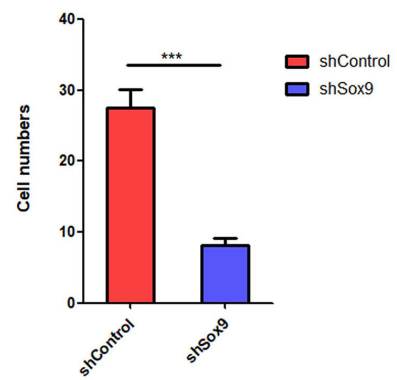

$\mathrm{F}$

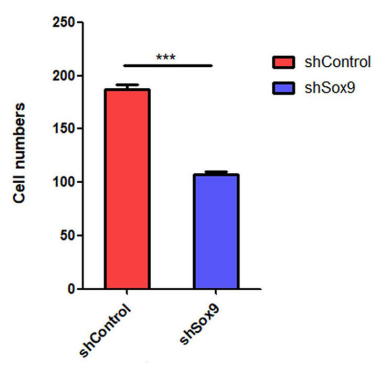

Fig. 2. The proliferation and migration abilities of HUC-MSCs transfected with shSOX9 or shControl. (A) CCK-8 Kit detected the proliferation of HUC-MSCs transfected with shSOX9 or shControl. (B) Count the cell number of HUC-MSCs transfected with shSOX9 or shControl at different times. (C) Cloning ability of HUC-MSCs transfected with shSOX9 or shControl. (D) Statistical analysis of the clone number according to figure subpart $\mathrm{C}\left(\mathrm{n}=3 ;{ }^{* * *} \mathrm{p}<0.001\right)$. (E) The migration of HUC-MSCs transfected with shSOX9 or shControl. (F) Statistical analysis of the migration number according to figure subpart $\mathrm{E}(\mathrm{n}=3 ; * * * \mathrm{p}<0.001)$.
A

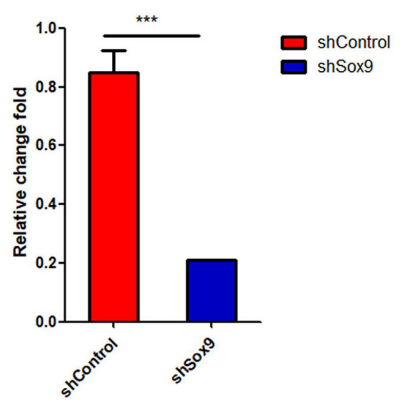

D

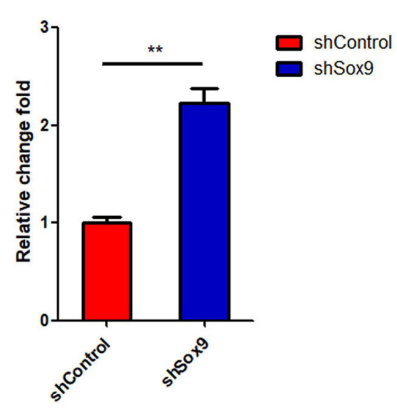

B

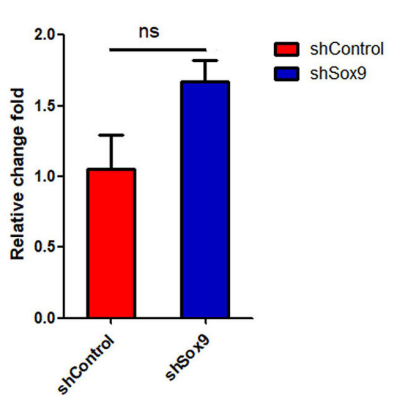

C

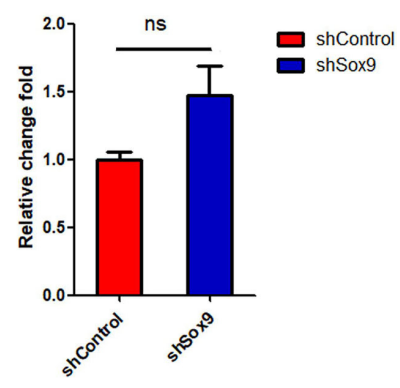

F
E

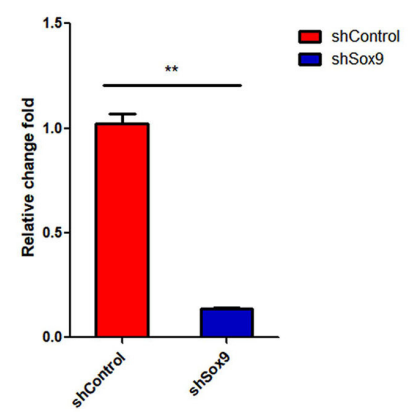

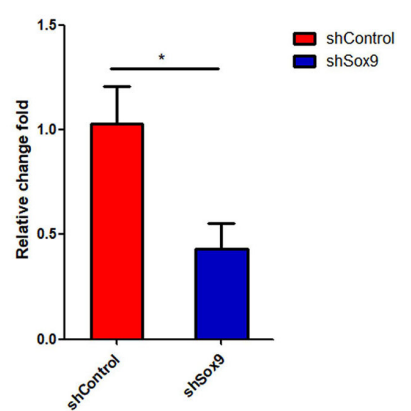

Fig. 3. The expression of cytokines and stemness related genes mRNA in HUC-MSCs transfected with shSOX9 or shControl. qRT-PCR was performed to detect the expression of IL-8 (A), IL-6 (B), GM-CSF (C), VEGF (D), OCT4 (E), SALL4 (F) $(\mathrm{n}=3 ; * \mathrm{p}<0.05 ; * * \mathrm{p}<0.01$; $* * * \mathrm{p}<0.001 ; \mathrm{ns}:$ no statistical difference). 
cord blood secreted growth factors, including GM-CSF and VEGF, by a cytokine protein array. We also measured the GM-CSF and VEGF mRNA expression in HUC-MSCs transfected with sh-control or shSOX9 by qRT-PCR and found that knockdown of SOX9 significantly enhanced VEGF mRNA expression and promoted GM-CSF mRNA expression with no significance (Fig. 3C and 3D). SOX9 interacted with Janus kinase 1 (JAK1) and regulated IL-6-JAK1-STAT3 signaling activation (40), and STAT3 signaling activation promoted the secretion of IL-6 cytokines and enhanced the stemness abilities of cells (41). Detailed molecular mechanisms involved in the secretion of cytokines of HUC-MSCs regulated by SOX9 need to further explore in the future. Knockdown of SOX9 also significantly inhibited the mRNA levels of the stemness-related genes OCT4 and SALL4 (Fig. 3E and 3F). These results indicated that SOX9 regulates the expression of cytokines and stemness-related genes in HUC-MSCs.
A

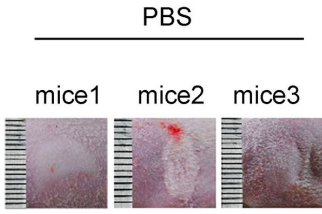

5 Day

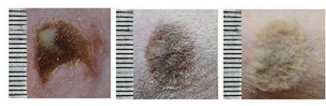

9Day

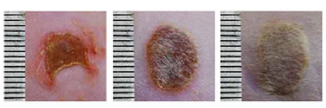

12Day

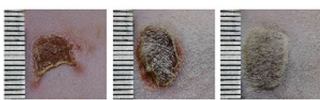

15Day

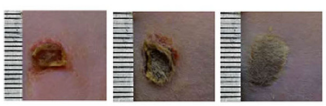

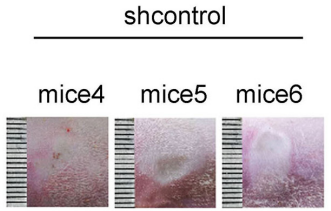
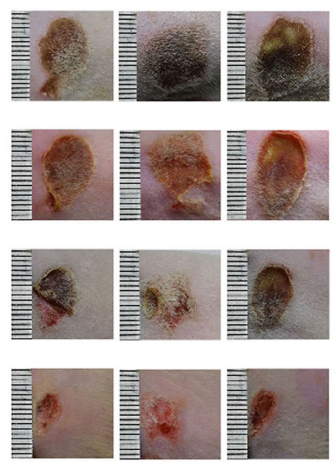

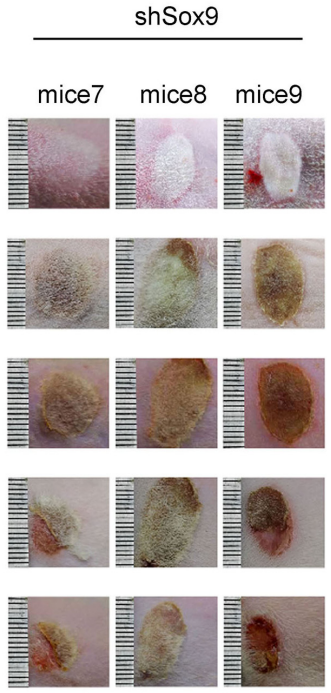

B

\section{Knockdown of SOX9 expression suppressed the pro-healing ability of HUC-MSCs in a skin wound model}

To explore the effects of SOX9 on the pro-healing ability of HUC-MSCs, we established a skin wound model in rats. As shown in our previous report (21), HUC-MSCs obviously promoted the skin repair of burn injury in rats (Fig. 4A). Knockdown of SOX9 expression in HUC-MSCs obviously decreased the pro-healing ability of HUC-MSCs in a skin wound model (Fig. 4A and 4B). We further evaluated the effects of SOX9 knockdown in HUC-MSCs on the repair of histological structure in the burn injury area. The results of histological evaluation of wounds post-infusion showed that the thickness of epidermal layer obviously increased in wounds treated with the HUC-MSCs transfected with sh-control (Fig. 5A and 5B), while epidermis repair of the wounds after treatment with PBS or the HUC-MSCs transfected with shSox9 was worse than that after treatment with the HUC-MSCs transfected with sh-control (Fig. 5A and 5B). The thickness of the dermal layer was not obviously different in these three groups (Fig. 5A and 5B). The results of Ki67 immunofluorescence
Fig. 4. Effects of knockdown SOX9 in HUC-MSCs on repairing in wound injury model of rat. (A) The scar of rat in wound injury model treated with PBS or HUC-MSCs transfected with shcontrol or shsox 9 at different timepoints day 1, 5, 9, $12,15(\mathrm{n}=3)$. (B) Statistical analysis of scar area in back of rat treated with PBS or HUC-MSCs transfected with shcontrol or shsox 9 at different timepoints from day $1,5,9,12,15$ $(n=3$, ns: no statistical difference). 
A
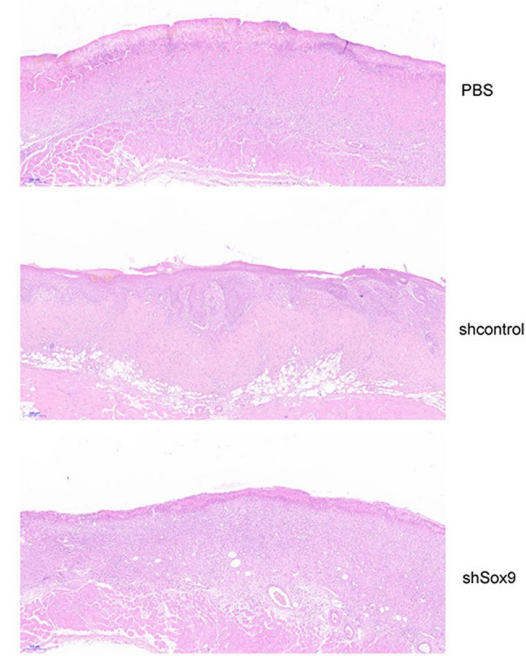

B

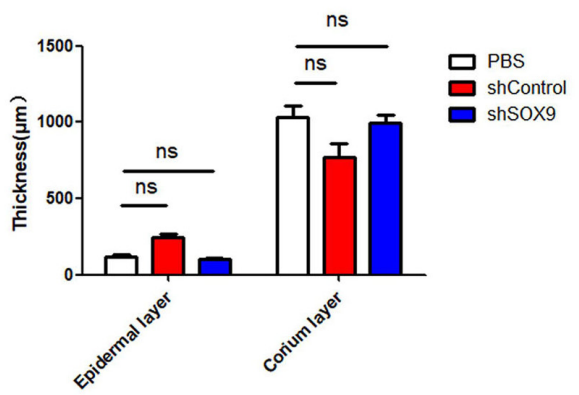

C
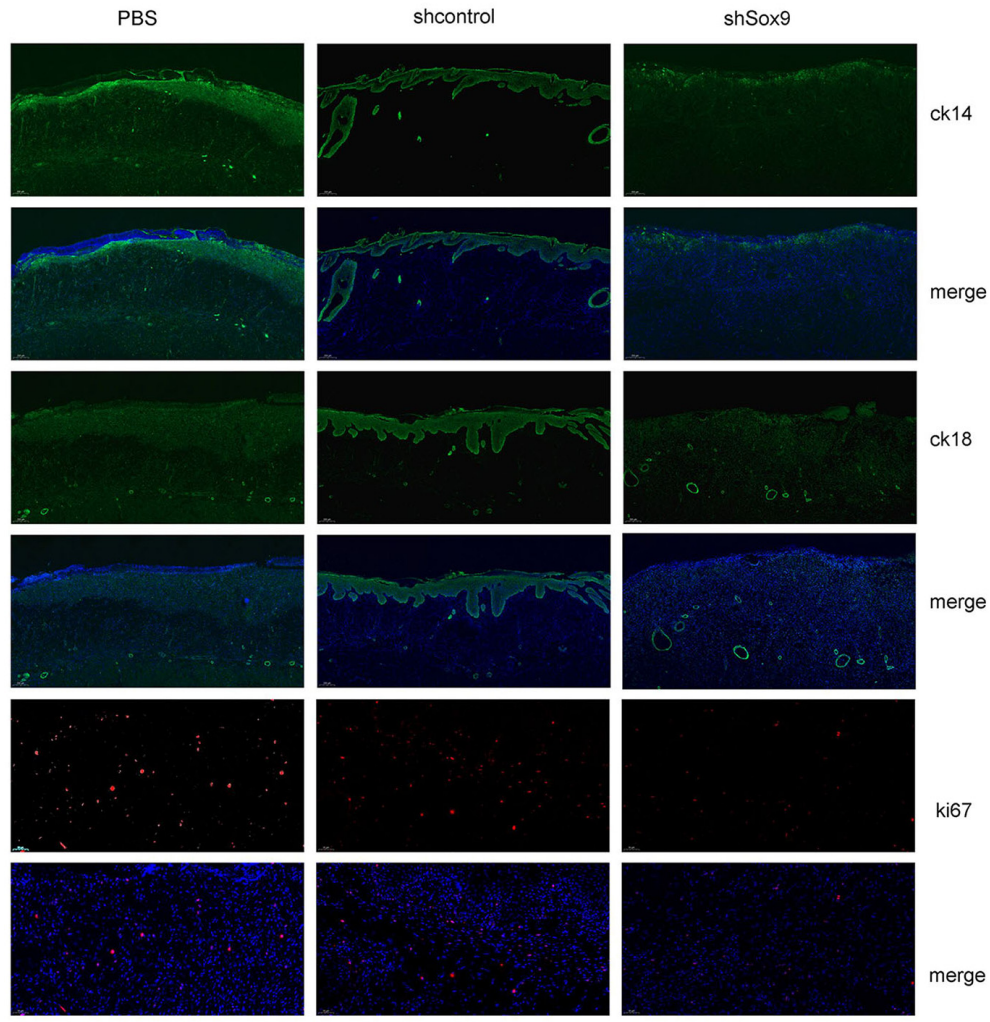

Fig. 5. Evaluate scar healing in rat treated with HUC-MSCs transfected with shSOX9 or shcontrol and PBS. HE staining the wound repair skin tissues of rat treated with PBS or HUC-MSCs transfected with shcontrol or shsox $9(n=3)$. (B) Statistical analysis the thickness of epidermis and dermis ( $n=3$, ns: no statistical difference). (C) Immunofluorescence staining the expression of CK14, CK18 and ki67 in the wound repair skin tissues of rat treated with PBS or HUC-MSCs transfected with shcontrol or shsox9 $(n=3)$.

staining showed that the HUC-MSCs transfected with sh-control had more Ki67-positive cells in the wound area than the HUC-MSCs transfected with shSox9 (Fig. 5C). We also measured the expression of epidermal markers, including CK14 and CK18, by immunofluorescence. The results suggested that epithelial repair in the wound injury of rats treated with the HUC-MSCs transfected with sh-control was more effective than that in the rats treated with PBS or the HUC-MSCs transfected with shSox9, and the accessory structures of skin, including hair follicles and glands, were also greater in the wound region of the rats treated with the HUC-MSCs transfected with sh-control (Fig. 5C). These results revealed that SOX9 influences the pro-healing ability of HUC-MSCs.

\section{Discussion}

HUC-MSCs, as prominent candidates in regenerative medicine, have the advantages of higher proliferation, faster self-renewal ability, lower immunogenicity and a more feasible harvest process than MSCs from other sources. Our previous studies revealed that HUC-MSC-derived exosomes might be a main mechanism of the HUC-MSC effects on skin wound healing $(21,22)$. These previous reports demonstrated that HUC-MSC-Ex-derived Wnt4 enhanced skin cell proliferation and migration and wound healing by activating Wnt $\beta$-catenin signaling and that HUC-MSC-Ex reduced cell apoptosis by activating AKT signaling (21). Subsequently, we found that HUC-MSC-Exs could control stem cell expansion after regenerative responses to prevent overcrowding and dysplasia by mechanisms by which HUC-MSC-Ex-derived 14-3-3 $\zeta$ promoted the formation of an inhibitory complex, YAP and p-LATS, to activate the Hippo pathway under high cell density conditions (22). In the present study, we found that knockdown of SOX9 expression inhibited the proliferation, mi- 
gration and prohealing ability of HUC-MSCs, and knockdown of SOX9 expression also influenced the expression of cytokines (IL-6 and IL-8), growth factors (GM-CSF and VEGF) and stemness-related genes (OCT4 and SALL). Whether knockdown of SOX9 expression influences the particle number of exosomes secreted from HUC-MSCs and influences the expression of the key proteins Wnt4, 14-3-3 $\zeta$, YAP and p-LATS, which play critical roles in repairing wound injury in skin, needs to be further studied. This study will provide guidance for modifying HUC-MSCs by bioengineering technology in regenerative medicine applications.

MSCs can secrete hundreds of factors, some of which, such as VEGF and IL-8, can stimulate angiogenesis, which is necessary for tumor growth and metastasis (38). Several studies have demonstrated that IL-8 participates in cancer cell survival, proliferation and invasion (42). Our results showed that knockdown of SOX9 expression in HUC-MSCs significantly inhibited the expression of IL-8 while upregulating the expression of VEGF. Several clinical studies have concluded that MSCs are safe with no obvious risk of tumorigenesis in treating injuries or other diseases (14, 43). Some studies also indicated that MSCs could promote the progression of tumors $(44,45)$. HUC-MSC clinical applications in regenerative medicine or other diseases are complex. Whether tumorigenesis of HUC-MSCs transplanted in humans might be closely related to the immune states of humans or the inflammatory microenvironment is unclear. Thus, applications of HUC-MSCs in clinical patients should be considered to evaluate the overall situation of patients comprehensively and individually.

SOX9 is a member of the sex-determining region $\mathrm{Y}$ (SRY)-related high mobility group (HMG)-box (SOX) transcription factor family (24). Stöckl et al. (46) found that Sox9 modulates cell survival and contributes to the commitment of mesenchymal stem cells to adipogenic or osteogenic differentiation lineages. Stöckl et al. (47) also proposed an osteogenic differentiation delaying role of Sox9 in rASCs. Overexpression of SOX9 in mesenchymal stem cells might strengthen the roles of MSCs in regenerative medicine. However, several studies have indicated that SOX9 expression is associated with tumor malignancy and tumorigenesis in various types of cancers $(48,49)$. Further exploration is needed to determine whether mesenchymal stem cells overexpressing SOX9 will be tumorigenic. In this study, we knocked down the expression of SOX9 and decreased the proliferation, migration and pro-healing ability of HUC-MSCs. Overexpression or knockdown of the key genes in HUC-MSCs to strengthen the function of HUC-MSCs in regenerative medicine or other diseases by bioengineering technology may be an important research direction in the future.

\section{Acknowledgments}

This study was supported by grants from the National Natural Science Foundation of China (No. 81702439, 81802446), project tsqn201909192 supported by Tai Shan Young Scholar Foundation of Shandong Province, project ZR2019BH050,ZR2020YQ59 supported by Shandong Provincial Natural Science Foundation; PhD Research Foundation of the Affiliated Hospital of Jining Medical University (No. 2018-BS-001, 2018-BS-013), project 202003 031182, 202003031183 supported by the Project of Medicine Health and Technology Development Plan of Shandong Province, Jining Medical University Teacher Research Support Fund (NO. JYFC2018FKJ035), the Miaopu Research of the Affiliated Hospital of Jining Medical University (No. MP-ZD-2020-005) and 2017 Technology Development Project of Shandong Medicine and Health Science (2017WSA10012), project CX2020081, CX2020042, CX2020035 supported by University Student Innovation Training Program of Jining Medical University.

\section{Potential Conflict of Interest}

The authors have no conflicting financial interest.

\section{References}

1. Friedenstein AJ. Precursor cells of mechanocytes. Int Rev Cytol 1976;47:327-359

2. Ding DC, Shyu WC, Lin SZ. Mesenchymal stem cells. Cell Transplant 2011;20:5-14

3. Erices A, Conget P, Minguell JJ. Mesenchymal progenitor cells in human umbilical cord blood. Br J Haematol 2000; 109:235-242

4. Zuk PA, Zhu M, Mizuno H, Huang J, Futrell JW, Katz AJ, Benhaim P, Lorenz HP, Hedrick MH. Multilineage cells from human adipose tissue: implications for cell-based therapies. Tissue Eng 2001;7:211-228

5. Lai D, Wang F, Dong Z, Zhang Q. Skin-derived mesenchymal stem cells help restore function to ovaries in a premature ovarian failure mouse model. PLoS One 2014;9: e98749

6. Ishige I, Nagamura-Inoue T, Honda MJ, Harnprasopwat R, Kido M, Sugimoto M, Nakauchi H, Tojo A. Comparison of mesenchymal stem cells derived from arterial, venous, and Wharton's jelly explants of human umbilical cord. Int J Hematol 2009;90:261-269

7. Yan K, Zhang R, Chen L, Chen F, Liu Y, Peng L, Sun H, Huang W, Sun C, Lv B, Li F, Cai Y, Tang Y, Zou Y, Du $M$, Qin L, Zhang H, Jiang X. Nitric oxide-mediated immunosuppressive effect of human amniotic membrane-derived mesenchymal stem cells on the viability and migra- 
tion of microglia. Brain Res 2014;1590:1-9

8. Mazini L, Rochette L, Admou B, Amal S, Malka G. Hopes and limits of adipose-derived stem cells (ADSCs) and mesenchymal stem cells (MSCs) in wound healing. Int J Mol Sci 2020;21:1306

9. Joshi M, B Patil P, He Z, Holgersson J, Olausson M, Sumitran-Holgersson S. Fetal liver-derived mesenchymal stromal cells augment engraftment of transplanted hepatocytes. Cytotherapy 2012;14:657-669

10. Ding DC, Chang YH, Shyu WC, Lin SZ. Human umbilical cord mesenchymal stem cells: a new era for stem cell therapy. Cell Transplant 2015;24:339-347

11. Chandravanshi B, Bhonde RR. Human umbilical cord-derived stem cells: isolation, characterization, differentiation, and application in treating diabetes. Crit Rev Biomed Eng 2018;46:399-412

12. Jiang Y, Zhu W, Zhu J, Wu L, Xu G, Liu X. Feasibility of delivering mesenchymal stem cells via catheter to the proximal end of the lesion artery in patients with stroke in the territory of the middle cerebral artery. Cell Transplant 2013;22:2291-2298

13. Fang TC, Pang CY, Chiu SC, Ding DC, Tsai RK. Renoprotective effect of human umbilical cord-derived mesenchymal stem cells in immunodeficient mice suffering from acute kidney injury. PLoS One 2012;7:e46504

14. Jin JL, Liu Z, Lu ZJ, Guan DN, Wang C, Chen ZB, Zhang J, Zhang WY, Wu JY, Xu Y. Safety and efficacy of umbilical cord mesenchymal stem cell therapy in hereditary spinocerebellar ataxia. Curr Neurovasc Res 2013;10:11-20

15. Batsali AK, Kastrinaki MC, Papadaki HA, Pontikoglou C. Mesenchymal stem cells derived from Wharton's Jelly of the umbilical cord: biological properties and emerging clinical applications. Curr Stem Cell Res Ther 2013;8:144-155

16. Kong D, Zhuang X, Wang D, Qu H, Jiang Y, Li X, Wu W, Xiao J, Liu X, Liu J, Li A, Wang J, Dou A, Wang Y, Sun J, Lv H, Zhang G, Zhang X, Chen S, Ni Y, Zheng C. Umbilical cord mesenchymal stem cell transfusion ameliorated hyperglycemia in patients with type 2 diabetes mellitus. Clin Lab 2014;60:1969-1976

17. Zhu L, Wang Z, Zheng X, Ding L, Han D, Yan H, Guo Z, Wang H. Haploidentical hematopoietic stem cell transplant with umbilical cord-derived multipotent mesenchymal cell infusion for the treatment of high-risk acute leukemia in children. Leuk Lymphoma 2015;56:1346-1352

18. Li T, Xia M, Gao Y, Chen Y, Xu Y. Human umbilical cord mesenchymal stem cells: an overview of their potential in cell-based therapy. Expert Opin Biol Ther 2015;15:12931306

19. Iqbal T, Saaiq M, Ali Z. Epidemiology and outcome of burns: early experience at the country's first national burns centre. Burns 2013;39:358-362

20. Blais M, Parenteau-Bareil R, Cadau S, Berthod F. Concise review: tissue-engineered skin and nerve regeneration in burn treatment. Stem Cells Transl Med 2013;2:545-551

21. Zhang B, Wang M, Gong A, Zhang X, Wu X, Zhu Y, Shi H, Wu L, Zhu W, Qian H, Xu W. HucMSC-exosome medi-
ated-Wnt4 signaling is required for cutaneous wound healing. Stem Cells 2015;33:2158-2168

22. Zhang B, Shi Y, Gong A, Pan Z, Shi H, Yang H, Fu H, Yan Y, Zhang X, Wang M, Zhu W, Qian H, Xu W. HucMSC exosome-delivered 14-3-3 $\zeta$ orchestrates self-control of the Wnt response via modulation of YAP during cutaneous regeneration. Stem Cells 2016;34:2485-2500

23. Zhang B, Wu X, Zhang X, Sun Y, Yan Y, Shi H, Zhu Y, Wu L, Pan Z, Zhu W, Qian H, Xu W. Human umbilical cord mesenchymal stem cell exosomes enhance angiogenesis through the Wnt $4 / \beta$-catenin pathway. Stem Cells Transl Med 2015;4:513-522

24. Lee YH, Saint-Jeannet JP. Sox9 function in craniofacial development and disease. Genesis 2011;49:200-208

25. Lefebvre V, Dumitriu B, Penzo-Méndez A, Han Y, Pallavi B. Control of cell fate and differentiation by Sry-related high-mobility-group box (Sox) transcription factors. Int J Biochem Cell Biol 2007;39:2195-2214

26. Jo A, Denduluri S, Zhang B, Wang Z, Yin L, Yan Z, Kang R, Shi LL, Mok J, Lee MJ, Haydon RC. The versatile functions of Sox9 in development, stem cells, and human diseases. Genes Dis 2014;1:149-161

27. Luanpitpong S, Li J, Manke A, Brundage K, Ellis E, McLaughlin SL, Angsutararux P, Chanthra N, Voronkova M, Chen YC, Wang L, Chanvorachote P, Pei M, Issaragrisil $\mathrm{S}$, Rojanasakul Y. SLUG is required for SOX9 stabilization and functions to promote cancer stem cells and metastasis in human lung carcinoma. Oncogene 2016;35:2824-2833

28. Voronkova MA, Luanpitpong S, Rojanasakul LW, Castranova V, Dinu CZ, Riedel H, Rojanasakul Y. SOX9 regulates cancer stem-like properties and metastatic potential of single-walled carbon nanotube-exposed cells. Sci Rep 2017;7: 11653

29. Azadniv M, Myers JR, McMurray HR, Guo N, Rock P, Coppage ML, Ashton J, Becker MW, Calvi LM, Liesveld JL. Bone marrow mesenchymal stromal cells from acute myelogenous leukemia patients demonstrate adipogenic differentiation propensity with implications for leukemia cell support. Leukemia 2020;34:391-403

30. Weissenberger $M$, Weissenberger $M H$, Gilbert F, Groll J, Evans CH, Steinert AF. Reduced hypertrophy in vitro after chondrogenic differentiation of adult human mesenchymal stem cells following adenoviral SOX9 gene delivery. BMC Musculoskelet Disord 2020;21:109

31. Yamada Y, Nakashima A, Doi S, Ishiuchi N, Kanai R, Miyasako K, Masaki T. Localization and maintenance of engrafted mesenchymal stem cells administered via renal artery in kidneys with ischemia-reperfusion injury. Int J Mol Sci 2021;22:4178

32. Qiao C, Xu W, Zhu W, Hu J, Qian H, Yin Q, Jiang R, Yan Y, Mao F, Yang H, Wang X, Chen Y. Human mesenchymal stem cells isolated from the umbilical cord. Cell Biol Int 2008;32:8-15

33. Bie Q, Song H, Chen X, Yang X, Shi S, Zhang L, Zhao R, Wei L, Zhang B, Xiong H, Zhang B. IL-17B/IL-17RB signaling cascade contributes to self-renewal and tumori- 
genesis of cancer stem cells by regulating Beclin-1 ubiquitination. Oncogene 2021;40:2200-2216

34. Wang YF, Dang HF, Luo X, Wang QQ, Gao C, Tian YX. Downregulation of SOX9 suppresses breast cancer cell proliferation and migration by regulating apoptosis and cell cycle arrest. Oncol Lett 2021;22:517

35. Gao J, Zhang JY, Li YH, Ren F. Decreased expression of SOX9 indicates a better prognosis and inhibits the growth of glioma cells by inducing cell cycle arrest. Int J Clin Exp Pathol 2015;8:10130-10138

36. Fu X, Liu G, Halim A, Ju Y, Luo Q, Song AG. Mesenchymal stem cell migration and tissue repair. Cells 2019;8:784

37. Zhong W, Zhu Z, Xu X, Zhang H, Xiong H, Li Q, Wei Y. Human bone marrow-derived mesenchymal stem cells promote the growth and drug-resistance of diffuse large B-cell lymphoma by secreting IL-6 and elevating IL-17A levels. J Exp Clin Cancer Res 2019;38:73

38. Wang J, Wang Y, Wang S, Cai J, Shi J, Sui X, Cao Y, Huang W, Chen X, Cai Z, Li H, Bardeesi AS, Zhang B, Liu M, Song W, Wang M, Xiang AP. Bone marrow-derived mesenchymal stem cell-secreted IL-8 promotes the angiogenesis and growth of colorectal cancer. Oncotarget 2015;6: 42825-42837

39. Liu CH, Hwang SM. Cytokine interactions in mesenchymal stem cells from cord blood. Cytokine 2005;32:270-279

40. Yang $\mathrm{H}$, Geng YH, Wang P, Yang H, Zhou YT, Zhang HQ, He HY, Fang WG, Tian XX. Extracellular ATP promotes breast cancer invasion and chemoresistance via SOX9 signaling. Oncogene 2020;39:5795-5810

41. Polak KL, Chernosky NM, Smigiel JM, Tamagno I, Jackson MW. Balancing STAT activity as a therapeutic strategy. Cancers (Basel) 2019;11:1716

42. Waugh DJ, Wilson C. The interleukin-8 pathway in cancer.
Clin Cancer Res 2008;14:6735-6741

43. Wang L, Wang L, Cong X, Liu G, Zhou J, Bai B, Li Y, Bai W, Li M, Ji H, Zhu D, Wu M, Liu Y. Human umbilical cord mesenchymal stem cell therapy for patients with active rheumatoid arthritis: safety and efficacy. Stem Cells Dev 2013;22:3192-3202

44. Zhu W, Xu W, Jiang R, Qian H, Chen M, Hu J, Cao W, Han C, Chen Y. Mesenchymal stem cells derived from bone marrow favor tumor cell growth in vivo. Exp Mol Pathol 2006;80:267-274

45. Chen B, Yu J, Wang Q, Zhao Y, Sun L, Xu C, Zhao X, Shen B, Wang $\mathrm{M}, \mathrm{Xu}$ W, Zhu W. Human bone marrow mesenchymal stem cells promote gastric cancer growth via regulating c-Myc. Stem Cells Int 2018;2018:9501747

46. Stöckl S, Bauer RJ, Bosserhoff AK, Göttl C, Grifka J, Grässel S. Sox9 modulates cell survival and adipogenic differentiation of multipotent adult rat mesenchymal stem cells. J Cell Sci 2013;126(Pt 13):2890-2902

47. Stöckl S, Göttl C, Grifka J, Grässel S. Sox9 modulates proliferation and expression of osteogenic markers of adiposederived stem cells (ASC). Cell Physiol Biochem 2013;31: 703-717

48. Wan YP, Xi M, He HC, Wan S, Hua W, Zen ZC, Liu YL, Zhou YL, Mo RJ, Zhuo YJ, Luo HW, Jiang FN, Zhong WD. Expression and clinical significance of SOX9 in renal cell carcinoma, bladder cancer and penile cancer. Oncol Res Treat 2017;40:15-20

49. Luo J, Bao YC, Ji XX, Chen B, Deng QF, Zhou SW. Corrigendum to "SPOP promotes SIRT2 degradation and suppresses non-small cell lung cancer cell growth" [Biochem. Biophys. Res. Commun. 483 (2017) 880-884]. Biochem Biophys Res Commun 2017;486:57 